\title{
A New Moving Frame for Trajectories with Non-Vanishing Angular Momentum
}

\author{
Kahraman Esen Özen ${ }^{1 *}$ and Murat Tosun ${ }^{2}$ \\ ${ }^{1}$ Sakarya, Turkey \\ ${ }^{2}$ Department of Mathematics, Faculty of Science and Arts, Sakarya University, Sakarya, Turkey \\ ${ }^{*}$ Corresponding author
}

\section{Article Info}

Keywords: Angular momentum, Kinematics of a particle, Moving frame, Plane and space curves, Slant helices. 2010 AMS: 70B99, 14 H50.

Received: 20 January 2021

Accepted: 26 March 2021

Available online: 30 April 2021

\begin{abstract}
The theory of curves has a very long history. Moving frames defined on curves are important parts of this theory. They have never lost their importance. A point particle of constant mass moving along a trajectory in space may be seen as a point of the trajectory. Therefore, there is a very close relationship between the differential geometry of the trajectory and the kinematics of the particle moving on it. One of the most important elements of the particle kinematics is the jerk vector of the moving particle. Recently, a new resolution of the jerk vector, along the tangential direction and two special radial directions, has been presented by Özen et al. (JTAM 57(2)(2019)). By means of these two special radial directions, we introduce a new moving frame for the trajectory of a moving particle with non vanishing angular momentum in this study. Then, according to this frame, some characterizations for the trajectory to be a rectifying curve, an osculating curve, a normal curve, a planar curve and a general helix are given. Also, slant helical trajectories are defined with respect to this frame. Afterwards, the necessary and sufficient conditions for the trajectory to be a slant helical trajectory (according to this frame) are obtained and some special cases of these trajectories are investigated. Moreover, we provide an illustrative numerical example to explain how this frame is constructed. This frame is a new contribution to the field and it may be useful in some specific applications of differential geometry, kinematics and robotics in the future.
\end{abstract}

\section{Introduction and preliminaries}

In differential geometry, the local theory of space curves plays an important role. The discovery of the Serret-Frenet formulas in 1847 was a milestone for the researchers who are interested in this theory. Despite its long history, it is still an issue of interest. The moving frames adapted to curves are useful tools for investigating this theory. From the discovery of the Serret-Frenet frame until now, many researchers have presented lots of interesting studies on this theory by using Serret-Frenet frame. Some of these studies can be found in [1]-[6].

From past to present, many researchers have developed new moving frames which have a common base vector with the Serret-Frenet frame. For example R. L. Bishop introduced parallel transport frame (or Bishop frame) in 1975 [7]. The first base vector of Serret-Frenet frame is included by this frame. A similar study was carried out by Y1lmaz and Turgut in 2010 by using the third base vector of the Serret-Frenet frame [8]. The authors called this frame as Type-2 Bishop frame. In 2019, a new version of Bishop frame was introduced by inspiring from these studies and by taking into consideration the second base vector of Serret-Frenet frame [9]. This frame is called Type-3 Bishop frame. There can be found many interesting studies such as [7]-[13] on various moving frames in the literature.

In Euclidean 3-space, a moving point particle of constant mass has a position vector according to the moving frame we are working on. By this way, any point on the trajectory can be represented by this particle. So, there is a very close relationship between the kinematics of a moving particle and the differential geometry of the trajectory which is the oriented curve traced out by this particle. As a result of this 
case, moving frames have been used as very useful tools to investigate the concepts on the kinematics of a moving particle such as position, velocity, acceleration and jerk vectors. Kinematics equations are used to determine the motion and to reach a desired position in robotics. To obtain an equation which includes all the position, velocity, acceleration and jerk, and to give the relationship between them provides various advantages for investigating some concepts in robotics such as minimum jerk trajectory generation. These facts and the importance of the position vector have motivated us in the process of preparing this study. We have constructed a new moving frame for the trajectories having non-vanishing angular momentum by using the own position vector of the moving particle. We expect that for the researchers studying on modern robotics, this moving frame will enable more convenient observation environment to understand the relationships between the basic concepts of kinematics and the basic concepts of inverse kinematics in the future.

Let us take into consideration the 3 -dimensional Euclidean space $E^{3}$ with the standard inner product:

$$
\langle\mathbf{K}, \mathbf{L}\rangle=k_{1} l_{1}+k_{2} l_{2}+k_{3} l_{3}
$$

where $\mathbf{K}=\left(k_{1}, k_{2}, k_{3}\right), \mathbf{L}=\left(l_{1}, l_{2}, l_{3}\right)$ are arbitrary vectors in this space. The norm of the vector $\mathbf{K}$ is expressed as $\|\mathbf{K}\|=\sqrt{\langle\mathbf{K}, \mathbf{K}\rangle}$. If a differentiable curve $\chi=\chi(s): I \subset \mathbb{R} \rightarrow E^{3}$ satisfies the equality $\left\|\frac{d \chi}{d s}\right\|=1$ for all $s \in I$, this curve is said to be a unit speed curve. In that case, $s$ is said to be arc-length parameter of this curve. A differentiable curve is called regular curve if its derivative is nonzero along the curve. Any regular curve can be reparameterized by the arc-length [14]. In the rest of this section, curves will be supposed to be regular and unit speed. Another thing that can be of importance is that the differentiation with respect to the arc-length parameter $s$ will be denoted by a dash throughout the present study.

It is well known that the unit tangent vector $\mathbf{T}=\delta^{\prime}$ of a given curve $\delta$ is determined uniquely. When the tangential components of two moving frames are common, these frames are called as equivalent frames. For example, Serret-Frenet frame and Bishop frame are equivalent frames.

Let $\left\{\mathbf{T}, \mathbf{N}_{\mathbf{1}}, \mathbf{N}_{\mathbf{2}}\right\}$ be a moving frame of a space curve which includes the unit tangent vector $\mathbf{T}$ and two normal unit vectors $\mathbf{N}_{\mathbf{1}}, \mathbf{N}_{\mathbf{2}}$. These three vectors together form a positively oriented orthonormal basis of the 3-dimensional Euclidean space $E^{3}$ attached to each point of the curve. Because of the orthonormality, the matrix form of derivative formulas are given as in the following:

$$
\left(\begin{array}{c}
\mathbf{T}^{\prime} \\
\mathbf{N}_{\mathbf{1}}{ }^{\prime} \\
\mathbf{N}_{\mathbf{2}}{ }^{\prime}
\end{array}\right)=\left(\begin{array}{ccc}
0 & k_{1} & k_{2} \\
-k_{1} & 0 & k_{3} \\
-k_{2} & -k_{3} & 0
\end{array}\right)\left(\begin{array}{c}
\mathbf{T} \\
\mathbf{N}_{\mathbf{1}} \\
\mathbf{N}_{\mathbf{2}}
\end{array}\right)
$$

where $k_{1}, k_{2}$, and $k_{3}$ are continuous coefficient functions. These coefficient functions are sufficient to characterize the geometry of the curve [15].

For the Serret-Frenet frame $k_{2}=0$. Also, $\mathbf{N}_{\mathbf{1}}, \mathbf{N}_{\mathbf{2}}, k_{1}$ and $k_{3}$ are commonly denoted by $\mathbf{N}, \mathbf{B}, \kappa$ and $\tau$, respectively. So, the derivative formulas take the following form:

$$
\left(\begin{array}{l}
\mathbf{T}^{\prime} \\
\mathbf{N}^{\prime} \\
\mathbf{B}^{\prime}
\end{array}\right)=\left(\begin{array}{ccc}
0 & \kappa & 0 \\
-\kappa & 0 & \tau \\
0 & -\tau & 0
\end{array}\right)\left(\begin{array}{l}
\mathbf{T} \\
\mathbf{N} \\
\mathbf{B}
\end{array}\right) .
$$

Here, the vector $\mathbf{N}=\frac{\delta^{\prime \prime}}{\left\|\delta^{\prime \prime}\right\|}$ and $\mathbf{B}=\mathbf{T} \wedge \mathbf{N}$ are specially called as principal normal vector and binormal vector of the given curve $\delta$, respectively. Also, the function $\kappa=\left\langle\mathbf{T}^{\prime}, \mathbf{N}\right\rangle$ is called as the curvature function, while the function $\tau=-\left\langle\mathbf{B}^{\prime}, \mathbf{N}\right\rangle$ is called as the torsion function [14]. In the rest of the study, we assume everywhere $\kappa \neq 0$.

This article is organized as follows. In Section 2, we explain how our frame is constructed and give the relation matrix between this frame and Serret-Frenet frame. Then, we obtain derivative formulas and complete the set of apparatus of this frame. Also, an illustrative example is given and the angular velocity vector is obtained for this frame. In Section 3, we obtain some necessary and sufficient conditions for the trajectory to be a rectifying curve, an osculating curve, a normal curve, a planar curve and a general helix. In Section 4, we define slant helical trajectories according to this frame. Then we give a characterization for these trajectories and investigate some special cases of these trajectories.

\section{Positional adapted frame}

Let us take into consideration a moving point particle of constant mass $m$ in space $E^{3}$. Choose an arbitrary fixed origin $O$ in space and denote by $\mathbf{x}$ the position vector of this particle at time $t$. Let the curve $\alpha=\alpha(s)$ be the unit speed parametrization of the trajectory of the particle where the arc-length $s$ of $\alpha$ corresponds to time $t$. Then, the unit tangent vector, velocity vector and linear momentum vector at the point $\alpha(s)$ (at time $t$ ) are given by

$$
\begin{aligned}
\mathbf{T}(s) & =\frac{d \mathbf{x}}{d s} \\
\mathbf{v}(t) & =\frac{d \mathbf{x}}{d t}=\left(\frac{d s}{d t}\right) \mathbf{T}(s) \\
\mathbf{p}(t) & =m \mathbf{v}(t)=m\left(\frac{d s}{d t}\right) \mathbf{T}(s),
\end{aligned}
$$

respectively [16]. On the other hand, we can write the following:

$$
\mathbf{x}=\langle\alpha(s), \mathbf{T}(s)\rangle \mathbf{T}(s)+\langle\alpha(s), \mathbf{N}(s)\rangle \mathbf{N}(s)+\langle\alpha(s), \mathbf{B}(s)\rangle \mathbf{B}(s)
$$


at the point $\alpha(s)$ (at time $t$ ) with respect to Serret-Frenet frame. By vector product of the position vector and the linear momentum vector at time $t$, the angular momentum vector of the particle about $O$ is obtained as follows:

$$
\mathbf{H}^{O}=m\langle\alpha(s), \mathbf{B}(s)\rangle\left(\frac{d s}{d t}\right) \mathbf{N}(s)-m\langle\alpha(s), \mathbf{N}(s)\rangle\left(\frac{d s}{d t}\right) \mathbf{B}(s) .
$$

In the rest of the study, we assume that angular momentum vector of the particle never vanishes. That is, we restrict ourselves to the trajectories having non-vanishing angular momentum. By this assumption, we ensure the coefficient functions $\langle\alpha(s), \mathbf{N}(s)\rangle$ and $\langle\alpha(s), \mathbf{B}(s)\rangle$ of the position vector not to be zero at the same time. In other words, we ensure that the tangent line never passes through the origin along the trajectory. Here we will use the special radial directions discussed in the study [17].

Now we return to the position vector in the equation (2.2). The opposite of this vector is given by

$$
-\mathbf{x}=\langle-\alpha(s), \mathbf{T}(s)\rangle \mathbf{T}(s)+\langle-\alpha(s), \mathbf{N}(s)\rangle \mathbf{N}(s)+\langle-\alpha(s), \mathbf{B}(s)\rangle \mathbf{B}(s) .
$$

The projections of this vector on the instantaneous osculating plane $\pi_{1}(s)$ and instantaneous rectifying plane $\pi_{2}(s)$ give us two vectors which will play an important role to construct our moving frame. These cases are explained in detail below:

The vector, whose starting point is $\alpha(s)$ and endpoint is the foot of the perpendicular that is from $O$ to $\pi_{1}(s)$, can be written as

$$
\mathbf{r}(s)=\langle-\alpha(s), \mathbf{T}(s)\rangle \mathbf{T}(s)+\langle-\alpha(s), \mathbf{N}(s)\rangle \mathbf{N}(s)
$$

and corresponds to the aforesaid projection on $\pi_{1}(s)$. On the other hand, the vector, whose starting point is $\alpha(s)$ and endpoint is the foot of the perpendicular that is from $O$ to $\pi_{2}(s)$, can be written as

$$
\mathbf{r}^{*}(s)=\langle-\alpha(s), \mathbf{T}(s)\rangle \mathbf{T}(s)+\langle-\alpha(s), \mathbf{B}(s)\rangle \mathbf{B}(s)
$$

and corresponds to the aforesaid projection on $\pi_{2}(s)$. Since the coefficient functions of the unit tangent vector are same in both equations (2.5) and (2.6), we can easily obtain the following vector

$$
\mathbf{r}(s)-\mathbf{r}^{*}(s)=\langle-\alpha(s), \mathbf{N}(s)\rangle \mathbf{N}(s)+\langle\alpha(s), \mathbf{B}(s)\rangle \mathbf{B}(s)
$$

whose starting point is $\alpha(s)$ and which lies on the instantaneous normal plane $\pi_{3}(s)$. Notice that the vector $\mathbf{r}(s)-\mathbf{r}^{*}(s)$ is equivalent to the vector whose starting point is the aforementioned foot on $\pi_{2}(s)$ and endpoint is the other aforementioned foot on $\pi_{1}(s)$ (see Figure 2.1).

Let us discuss on the determination of the unit vector in direction of the vector $\mathbf{r}(s)-\mathbf{r}^{*}(s)$. When both the planes $\pi_{1}(s)$ and $\pi_{2}(s)$ do not contain the origin, the foots are distinct from each other and from the origin. Thus two distinct points (foots) generate the non-zero vector $\mathbf{r}(s)-\mathbf{r}^{*}(s)$. So, the desired unit vector can be immediately determined. If only one of the planes $\pi_{1}(s)$ and $\pi_{2}(s)$ passes through the origin, the foot of the perpendicular on the plane, containing origin, is taken as the origin. Of course, the other foot on the other plane is distinct from the origin. Then, the desired unit vector is obtained similarly. The case both the planes $\pi_{1}(s)$ and $\pi_{2}(s)$ contain the origin simultaneously causes not to be determined of the desired unit vector since the both of the aforementioned foots correspond to the origin in this case. That situation occurs only when the tangent line passes through the origin. Fortunately, our assumption, on the angular momentum vector, averts this case. Let the unit vector in direction of the vector $\mathbf{r}(s)-\mathbf{r}^{*}(s)$ be shown with $\mathbf{Y}(s)$. That is,

$$
\mathbf{Y}(s)=\frac{\mathbf{r}(s)-\mathbf{r}^{*}(s)}{\left\|\mathbf{r}(s)-\mathbf{r}^{*}(s)\right\|}=\frac{\langle-\alpha(s), \mathbf{N}(s)\rangle}{\sqrt{\langle\alpha(s), \mathbf{N}(s)\rangle^{2}+\langle\alpha(s), \mathbf{B}(s)\rangle^{2}}} \mathbf{N}(s)+\frac{\langle\alpha(s), \mathbf{B}(s)\rangle}{\sqrt{\langle\alpha(s), \mathbf{N}(s)\rangle^{2}+\langle\alpha(s), \mathbf{B}(s)\rangle^{2}}} \mathbf{B}(s) .
$$

By vector product the vectors $\mathbf{Y}(s)$ and $\mathbf{T}(s)$, we can obtain the another basis vector. We denote it by $\mathbf{M}(s)$. Then, we have

$$
\mathbf{M}(s)=\mathbf{Y}(s) \wedge \mathbf{T}(s)=\frac{\langle\alpha(s), \mathbf{B}(s)\rangle}{\sqrt{\langle\alpha(s), \mathbf{N}(s)\rangle^{2}+\langle\alpha(s), \mathbf{B}(s)\rangle^{2}}} \mathbf{N}(s)+\frac{\langle\alpha(s), \mathbf{N}(s)\rangle}{\sqrt{\langle\alpha(s), \mathbf{N}(s)\rangle^{2}+\langle\alpha(s), \mathbf{B}(s)\rangle^{2}}} \mathbf{B}(s) .
$$

This completes the positively oriented orthonormal moving frame $\{\mathbf{T}(s), \mathbf{M}(s), \mathbf{Y}(s)\}$.

Since the vectors $\mathbf{N}(s), \mathbf{B}(s), \mathbf{M}(s)$ and $\mathbf{Y}(s)$ lie on the instantaneous normal plane $\pi_{3}(s)$, there is a relation between the Serret-Frenet frame and this frame as in the following:

$$
\left(\begin{array}{c}
\mathbf{T}(s) \\
\mathbf{M}(s) \\
\mathbf{Y}(s)
\end{array}\right)=\left(\begin{array}{ccc}
1 & 0 & 0 \\
0 & \cos \Omega(s) & -\sin \Omega(s) \\
0 & \sin \Omega(s) & \cos \Omega(s)
\end{array}\right)\left(\begin{array}{l}
\mathbf{T}(s) \\
\mathbf{N}(s) \\
\mathbf{B}(s)
\end{array}\right)
$$

where $\Omega(s)$ is the angle between the vectors $\mathbf{B}(s)$ and $\mathbf{Y}(s)$ which is positively oriented from $\mathbf{B}(s)$ to $\mathbf{Y}(s)$ (see Figure 2.1).

By using the matrix equations (1.3) and (2.10), we can easily write

$$
\begin{aligned}
\mathbf{M}^{\prime}(s) & =(\cos \Omega(s) \mathbf{N}(s)-\sin \Omega(s) \mathbf{B}(s))^{\prime} \\
& =-\Omega^{\prime}(s) \sin \Omega(s) \mathbf{N}(s)+\cos \Omega(s)(-\kappa(s) \mathbf{T}(s)+\tau(s) \mathbf{B}(s))-\Omega^{\prime}(s) \cos \Omega(s) \mathbf{B}(s)+\tau(s) \sin \Omega(s) \mathbf{N}(s) \\
& =-\kappa(s) \cos \Omega(s) \mathbf{T}(s)+\left(\tau(s)-\Omega^{\prime}(s)\right)[\sin \Omega(s) \mathbf{N}(s)+\cos \Omega(s) \mathbf{B}(s)] \\
& =(-\kappa(s) \cos \Omega(s)) \mathbf{T}(s)+\left(\tau(s)-\Omega^{\prime}(s)\right) \mathbf{Y}(s)
\end{aligned}
$$


and

$$
\begin{aligned}
\mathbf{Y}^{\prime}(s) & =(\sin \Omega(s) \mathbf{N}(s)+\cos \Omega(s) \mathbf{B}(s))^{\prime} \\
& =-\Omega^{\prime}(s) \cos \Omega(s) \mathbf{N}(s)+\sin \Omega(s)(-\kappa(s) \mathbf{T}(s)+\tau(s) \mathbf{B}(s))-\Omega^{\prime}(s) \sin \Omega(s) \mathbf{B}(s)-\tau(s) \cos \Omega(s) \mathbf{N}(s) \\
& =-\kappa(s) \sin \Omega(s) \mathbf{T}(s)+\left(\Omega^{\prime}(s)-\tau(s)\right)[\cos \Omega(s) \mathbf{N}(s)-\sin \Omega(s) \mathbf{B}(s)] \\
& =(-\kappa(s) \sin \Omega(s)) \mathbf{T}(s)+\left(\Omega^{\prime}(s)-\tau(s)\right) \mathbf{M}(s) .
\end{aligned}
$$

Then, differentiating the vector $\mathbf{M}(s) \wedge \mathbf{Y}(s)$ yields the following:

$$
\begin{aligned}
\mathbf{T}^{\prime}(s) & =(\mathbf{M}(s) \wedge \mathbf{Y}(s))^{\prime} \\
& =\mathbf{M}^{\prime}(s) \wedge \mathbf{Y}(s)+\mathbf{M}(s) \wedge \mathbf{Y}^{\prime}(s) \\
& =\left[(-\kappa(s) \cos \Omega(s)) \mathbf{T}(s)+\left(\tau(s)-\Omega^{\prime}(s)\right) \mathbf{Y}(s)\right] \wedge \mathbf{Y}(s)+\mathbf{M}(s) \wedge\left[(-\kappa(s) \sin \Omega(s)) \mathbf{T}(s)+\left(\Omega^{\prime}(s)-\tau(s)\right) \mathbf{M}(s)\right] \\
& =(-\kappa(s) \cos \Omega(s))(\mathbf{T}(s) \wedge \mathbf{Y}(s))+(-\kappa(s) \sin \Omega(s))(\mathbf{M}(s) \wedge \mathbf{T}(s)) \\
& =(\kappa(s) \cos \Omega(s)) \mathbf{M}(s)+(\kappa(s) \sin \Omega(s)) \mathbf{Y}(s)
\end{aligned}
$$

Thus, the matrix form of the derivative formulas is immediately given by

$$
\left(\begin{array}{c}
\mathbf{T}^{\prime}(s) \\
\mathbf{M}^{\prime}(s) \\
\mathbf{Y}^{\prime}(s)
\end{array}\right)=\left(\begin{array}{ccc}
0 & k_{1}(s) & k_{2}(s) \\
-k_{1}(s) & 0 & k_{3}(s) \\
-k_{2}(s) & -k_{3}(s) & 0
\end{array}\right)\left(\begin{array}{c}
\mathbf{T}(s) \\
\mathbf{M}(s) \\
\mathbf{Y}(s)
\end{array}\right)
$$

where

$$
\begin{aligned}
k_{1}(s) & =\kappa(s) \cos \Omega(s) \\
k_{2}(s) & =\kappa(s) \sin \Omega(s) \\
k_{3}(s) & =\tau(s)-\Omega^{\prime}(s) \\
\tan \Omega(s) & =\frac{k_{2}(s)}{k_{1}(s)} \\
\kappa(s) & =\sqrt{k_{1}^{2}(s)+k_{2}^{2}(s) .}
\end{aligned}
$$

Based on the relationship of the frame $\{\mathbf{T}(s), \mathbf{M}(s), \mathbf{Y}(s)\}$ to the position vector, we call it as "Positional Adapted Frame". For convenience, we will use the abbreviation PAF instead of the "Positional Adapted Frame" in the rest of the study. Also, we call the set $\left\{\mathbf{T}(s), \mathbf{M}(s), \mathbf{Y}(s), k_{1}(s), k_{2}(s), k_{3}(s)\right\}$ as PAF apparatus of the curve $\alpha=\alpha(s)$.
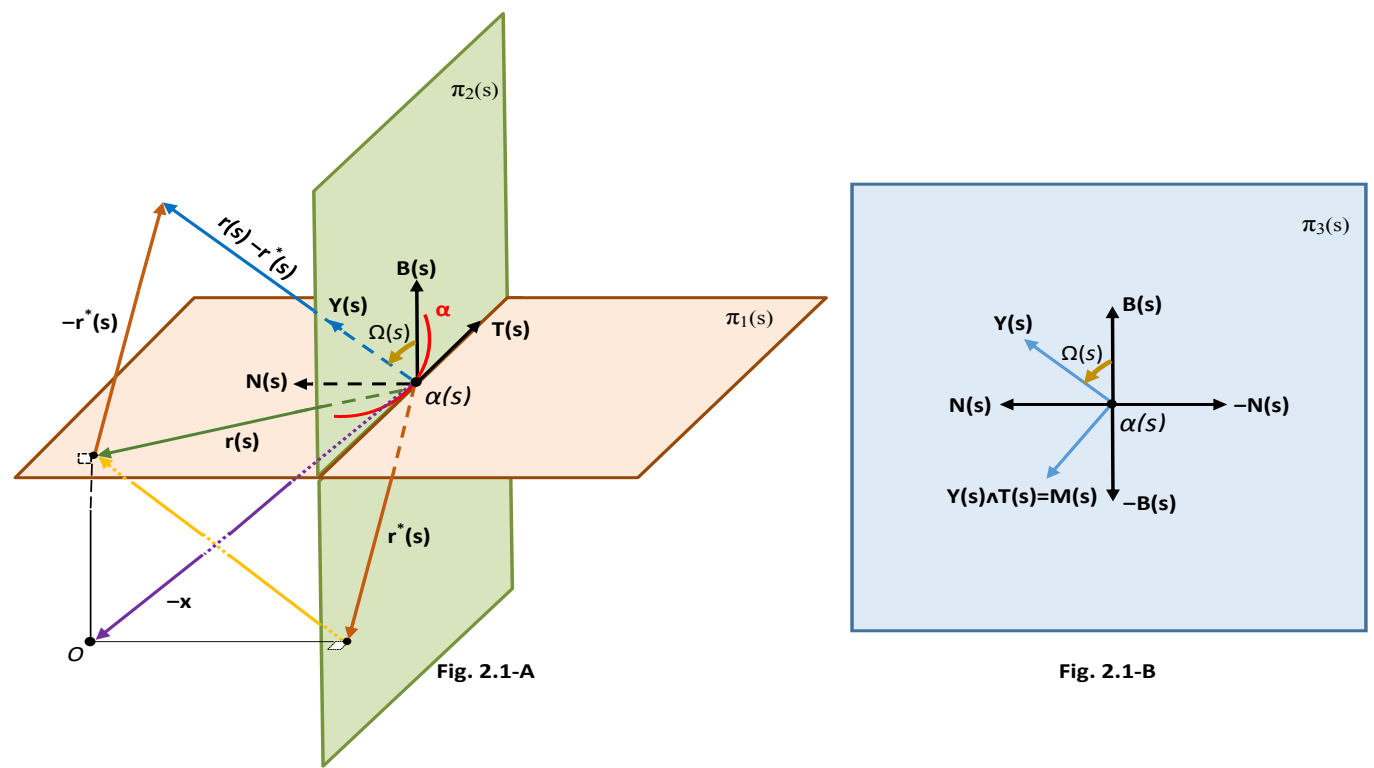

Figure 2.1: An illustration for explaining the construction of PAF

It is well known from linear algebra that a vector can be written uniquely in terms of the basis vectors. This basic knowledge yields the equations

$$
\begin{aligned}
\sin \Omega(s) & =\frac{-\langle\alpha(s), \mathbf{N}(s)\rangle}{\sqrt{\langle\alpha(s), \mathbf{N}(s)\rangle^{2}+\langle\alpha(s), \mathbf{B}(s)\rangle^{2}}} \\
\cos \Omega(s) & =\frac{\langle\alpha(s), \mathbf{B}(s)\rangle}{\sqrt{\langle\alpha(s), \mathbf{N}(s)\rangle^{2}+\langle\alpha(s), \mathbf{B}(s)\rangle^{2}}}
\end{aligned}
$$


if we compare the equation (2.10) with the equations (2.8) and (2.9). Then, we get

$$
\tan \Omega(s)=-\frac{\langle\alpha(s), \mathbf{N}(s)\rangle}{\langle\alpha(s), \mathbf{B}(s)\rangle}
$$

Considering the information given in (2.13), (2.14) and (2.15), the rotation angle $\Omega(s)$ is determined as follows:

$$
\Omega(s)=\left\{\begin{array}{c}
\arctan \left(-\frac{\langle\alpha(s), \mathbf{N}(s)\rangle}{\alpha \alpha(s), \mathbf{B}(s)\rangle}\right) \text { if }\langle\alpha(s), \mathbf{B}(s)\rangle>0 \\
\arctan \left(-\frac{\langle\alpha(s), \mathbf{N}(s)\rangle}{\langle\alpha(s), \mathbf{B}(s)\rangle}\right)+\pi \text { if }\langle\alpha(s), \mathbf{B}(s)\rangle<0 \\
-\frac{\pi}{2} \text { if }\langle\alpha(s), \mathbf{B}(s)\rangle=0,\langle\alpha(s), \mathbf{N}(s)\rangle>0 \\
\frac{\pi}{2} \text { if }\langle\alpha(s), \mathbf{B}(s)\rangle=0,\langle\alpha(s), \mathbf{N}(s)\rangle<0 .
\end{array}\right.
$$

One can easily complete the calculations by the aid Mathematica program. In the case $\langle\alpha(s), \mathbf{B}(s)\rangle=0,\langle\alpha(s), \mathbf{N}(s)\rangle>0$, the PAF apparatus $\left\{\mathbf{T}(s), \mathbf{M}(s), \mathbf{Y}(s), k_{1}(s), k_{2}(s), k_{3}(s)\right\}$ correspond to $\{\mathbf{T}(s), \mathbf{B}(s),-\mathbf{N}(s), 0,-\kappa(s), \tau(s)\}$. Similarly, when $\langle\alpha(s), \mathbf{B}(s)\rangle=0,\langle\alpha(s), \mathbf{N}(s)\rangle<0$, the PAF apparatus $\left\{\mathbf{T}(s), \mathbf{M}(s), \mathbf{Y}(s), k_{1}(s), k_{2}(s), k_{3}(s)\right\}$ correspond to $\{\mathbf{T}(s),-\mathbf{B}(s), \mathbf{N}(s), 0, \kappa(s), \tau(s)\}$.

Now, we will obtain the angular velocity vector for PAF. A better insight into the structure of the derivative formulas, given in (2.11), is presented by means of the angular velocity vector $\omega(s)$. The evolution of $\operatorname{PAF}\{\mathbf{T}(s), \mathbf{M}(s), \mathbf{Y}(s)\}$ is specified by its angular velocity via following relations:

$$
\begin{aligned}
\mathbf{T}^{\prime}(s) & =\omega(s) \wedge \mathbf{T}(s) \\
\mathbf{M}^{\prime}(s) & =\omega(s) \wedge \mathbf{M}(s) \\
\mathbf{Y}^{\prime}(s) & =\omega(s) \wedge \mathbf{Y}(s) .
\end{aligned}
$$

Let us determine the vector $\omega(s)$. Suppose that it is written with respect to PAF as in the following:

$$
\omega(s)=a(s) \mathbf{T}(s)+b(s) \mathbf{M}(s)+c(s) \mathbf{Y}(s)
$$

where $a(s), b(s)$ and $c(s)$ are real-valued functions of the parameter $s$. Then (2.17) becomes

$$
\begin{aligned}
\mathbf{T}^{\prime}(s) & =-b(s) \mathbf{Y}(s)+c(s) \mathbf{M}(s) \\
\mathbf{M}^{\prime}(s) & =a(s) \mathbf{Y}(s)-c(s) \mathbf{T}(s) \\
\mathbf{Y}^{\prime}(s) & =-a(s) \mathbf{M}(s)+b(s) \mathbf{T}(s)
\end{aligned}
$$

By comparing (2.11) with (2.18) we get

$$
\begin{aligned}
a & =k_{3} \\
b & =-k_{2} \\
c & =k_{1} .
\end{aligned}
$$

Therefore, the angular velocity vector is given by

$$
\omega(s)=k_{3}(s) \mathbf{T}(s)-k_{2}(s) \mathbf{M}(s)+k_{1}(s) \mathbf{Y}(s)
$$

for PAF.

Example 2.1. In $E^{3}$, assume that a point particle $P$ of constant mass moves on the trajectory $\delta:(0, k) \rightarrow E^{3}, \delta(s)=\left(8 \cos \frac{s}{17}, 8 \sin \frac{s}{17}, 15 \frac{s}{17}\right)$, which is a unit speed curve, where $k$ is a positive real constant. See the trajectory $\delta=\delta(s)$ in Figure 2.2. The Serret-Frenet apparatus of this trajectory are expressed as in [18]:

$$
\begin{aligned}
\mathbf{T}(s) & =\left(-\frac{8}{17} \sin \frac{s}{17}, \frac{8}{17} \cos \frac{s}{17}, \frac{15}{17}\right) \\
\mathbf{N}(s) & =\left(-\cos \frac{s}{17},-\sin \frac{s}{17}, 0\right) \\
\mathbf{B}(s) & =\left(\frac{15}{17} \sin \frac{s}{17},-\frac{15}{17} \cos \frac{s}{17}, \frac{8}{17}\right) \\
\kappa(s) & =\frac{8}{289} \\
\tau(s) & =\frac{15}{289} .
\end{aligned}
$$




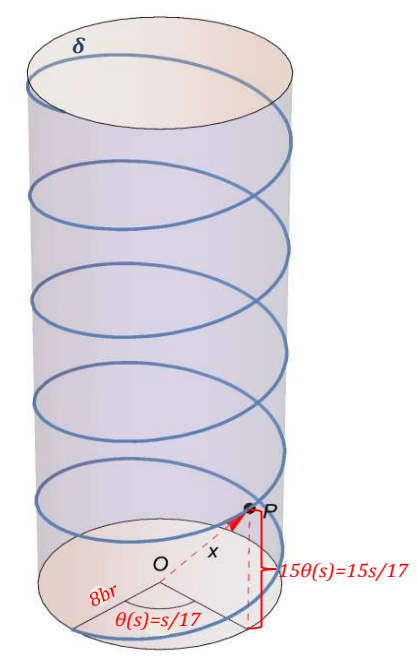

Figure 2.2: An illustration for the trajectory given in Example 2.1

Then, $\langle\boldsymbol{\delta}(s), \mathbf{N}(s)\rangle=-8$ and $\langle\boldsymbol{\delta}(s), \mathbf{B}(s)\rangle=\frac{120}{289}$ s are easily obtained. Since $\langle\boldsymbol{\delta}(s), \mathbf{B}(s)\rangle>0$ for all $s \in(0, k)$, we get $\Omega(s)=\arctan \left(\frac{289}{15 s}\right)$. The above information gives us the PAF apparatus as follows:

$$
\begin{aligned}
& \mathbf{T}(s)=\left(\frac{-8}{17} \sin \frac{s}{17}, \frac{8}{17} \cos \frac{s}{17}, \frac{15}{17}\right) \\
& \mathbf{M}(s)=\left(-\cos \left(\arctan \left(\frac{289}{15 s}\right)\right) \cos \frac{s}{17}-\frac{15}{17} \sin \left(\arctan \left(\frac{289}{15 s}\right)\right) \sin \frac{s}{17},-\cos \left(\arctan \left(\frac{289}{15 s}\right)\right) \sin \frac{s}{17}+\frac{15}{17} \sin \left(\arctan \left(\frac{289}{15 s}\right)\right) \cos \frac{s}{17}, \frac{-8}{17} \sin \left(\arctan \left(\frac{289}{15 s}\right)\right)\right) \\
& \mathbf{Y}(s)=\left(-\sin \left(\arctan \left(\frac{289}{15 s}\right)\right) \cos \frac{s}{17}+\frac{15}{17} \cos \left(\arctan \left(\frac{289}{15 s}\right)\right) \sin \frac{s}{17},-\sin \left(\arctan \left(\frac{289}{15 s}\right)\right) \sin \frac{s}{17}-\frac{15}{17} \cos \left(\arctan \left(\frac{289}{15 s}\right)\right) \cos \frac{s}{17}, \frac{8}{17} \cos \left(\arctan \left(\frac{289}{15 s}\right)\right)\right) \\
& k_{1}(s)=\frac{8}{289} \cos \left(\arctan \left(\frac{289}{15 s}\right)\right) \\
& k_{2}(s)=\frac{8}{289} \sin \left(\arctan \left(\frac{289}{15 s}\right)\right) \\
& k_{3}(s)=\frac{15}{289}+\frac{4335}{83521+225 s^{2}} \\
& \text { in the light of the equations }(2.10) \text { and (2.12). }
\end{aligned}
$$

\section{Some characterizations for the special cases of the trajectory}

In this section, we obtain some conditions including the PAF apparatus for the trajectory to be a rectifying curve, an osculating curve, a normal curve, a planar curve and a general helix in $E^{3}$.

It is not difficult to see

$$
\begin{aligned}
& k_{1}(s)=\kappa(s)\left(\frac{\langle\alpha(s), \mathbf{B}(s)\rangle}{\sqrt{\langle\alpha(s), \mathbf{N}(s)\rangle^{2}+\langle\alpha(s), \mathbf{B}(s)\rangle^{2}}}\right) \\
& k_{2}(s)=\kappa(s)\left(\frac{-\langle\alpha(s), \mathbf{N}(s)\rangle}{\sqrt{\langle\alpha(s), \mathbf{N}(s)\rangle^{2}+\langle\alpha(s), \mathbf{B}(s)\rangle^{2}}}\right)
\end{aligned}
$$

considering the above derivation. Then, by keeping $\kappa \neq 0$ and the assumption related to angular momentum in mind, it can be said that $k_{1}(s)$ and $k_{2}(s)$ can not be equal to zero at the same time and they verify the propositions

$$
\begin{array}{lll}
k_{1}(s)=0 & \Leftrightarrow & \langle\alpha(s), \mathbf{B}(s)\rangle=0 \\
k_{2}(s)=0 & \Leftrightarrow & \langle\alpha(s), \mathbf{N}(s)\rangle=0 .
\end{array}
$$

So, the equation $k_{1}(s)\langle\alpha(s), \mathbf{N}(s)\rangle+k_{2}(s)\langle\alpha(s), \mathbf{B}(s)\rangle=0$ is satisfied if $k_{1}(s)=0$. Also, from the above propositions and the equations $(2.12)_{4}$ and (2.15) we have the following:

$$
\frac{k_{2}(s)}{k_{1}(s)}=-\frac{\langle\alpha(s), \mathbf{N}(s)\rangle}{\langle\alpha(s), \mathbf{B}(s)\rangle}
$$

where $k_{1}(s) \neq 0$. Thus, the aforementioned equation

$$
k_{1}(s)\langle\alpha(s), \mathbf{N}(s)\rangle+k_{2}(s)\langle\alpha(s), \mathbf{B}(s)\rangle=0
$$

is satisfied for all the $s$ values of parameter.

A curve $\alpha=\alpha(s)$ is called as osculating curve (rectifying curve or normal curve) if its position vector always lies in its osculating plane (rectifying plane or normal plane) (see [4]-[6] for more details). Considering these definitions, the following theorems can be given. 
Theorem 3.1. In $E^{3}$, suppose that a point particle of constant mass moves on its trajectory having non-vanishing angular momentum. Let $\alpha=\alpha(s)$ be the unit speed parametrization of the trajectory with $\kappa \neq 0$. Then, $\alpha$ is a rectifying curve if and only if $k_{2}=0$.

Proof. Let $\alpha=\alpha(s)$ be the unit speed parametrization of the trajectory with $\kappa \neq 0$. Assume that $\alpha$ is a rectifying curve. Then its position vector always lies on its rectifying plane. So, $\langle\alpha(s), \mathbf{N}(s)\rangle=0$ for all the values $s$ of the arc-length parameter. Taking into consideration the equation (3.2), we get

$$
k_{2}(s)\langle\alpha(s), \mathbf{B}(s)\rangle=0
$$

for all $s$. Due to the non-vanishing angular momentum, the tangent line never passes through the origin $O$. Thus, $\langle\alpha(s), \mathbf{B}(s)\rangle$ never vanishes along the rectifying trajectory $\alpha$. Using this information in the equation (3.3) completes the first part of the proof.

On the contrary, assume that $k_{2}=0$. From (3.2), we get

$$
\forall s, \quad k_{1}(s)\langle\alpha(s), \mathbf{N}(s)\rangle=0 .
$$

We know that $k_{1}(s)$ and $k_{2}(s)$ can not be equal to zero at the same time. So, we can ensure that $k_{1}(s)$ never vanishes. This yields the following

$$
\forall s,\langle\alpha(s), \mathbf{N}(s)\rangle=0
$$

which means that $\alpha=\alpha(s)$ is a rectifying curve.

Theorem 3.2. In $E^{3}$, assume that a point particle of constant mass moves on its trajectory having non-vanishing angular momentum. Let $\alpha=\alpha(s)$ be the unit speed parametrization of the trajectory with $\kappa \neq 0$. In this case, $\alpha$ is an osculating curve if and only if $k_{1}=0$.

Proof. Let $\alpha=\alpha(s)$ be the unit speed parametrization of the trajectory with $\kappa \neq 0$. Suppose that $\alpha$ is an osculating curve. In that case, its position vector always lies on its osculating plane. Thus, $\langle\alpha(s), \mathbf{B}(s)\rangle=0$ for all the values $s$ of the arc-length parameter. Considering the equation (3.2), we get

$$
k_{1}(s)\langle\alpha(s), \mathbf{N}(s)\rangle=0
$$

for all $s$. Similarly previous proof, in the light of non-vanishing angular momentum we can ensure that the tangent line never passes through the origin $O$. So, $\langle\alpha(s), \mathbf{N}(s)\rangle$ never vanishes along the osculating trajectory $\alpha$. Then we conclude

$$
\forall s, \quad k_{1}(s)=0
$$

and finish the first part of the proof.

On the contrary, suppose that $k_{1}=0$. Then from the equation (3.2)

$$
\forall s, \quad k_{2}(s)\langle\alpha(s), \mathbf{B}(s)\rangle=0
$$

can be written. Since $k_{1}(s)$ and $k_{2}(s)$ are not equal to zero at the same time, we can say

$$
\forall s, \quad k_{2}(s) \neq 0 .
$$

This gives us the following

$$
\forall s,\langle\alpha(s), \mathbf{B}(s)\rangle=0
$$

which means that $\alpha=\alpha(s)$ is an osculating curve.

Theorem 3.3. In $E^{3}$, assume that a point particle of constant mass moves on its trajectory having non-vanishing angular momentum. Let $\alpha=\alpha(s)$ be the unit speed parametrization of the trajectory with $\kappa \neq 0$. Then the following properties hold:

1. If $\alpha$ is a normal curve, then the differential equation $k_{2} k_{1}{ }^{\prime}-k_{1} k_{2}{ }^{\prime}-k_{3}\left(k_{1}{ }^{2}+k_{2}{ }^{2}\right)-\Omega^{\prime}\left(k_{1}{ }^{2}+k_{2}{ }^{2}\right)=0$ is satisfied along $\alpha$.

2. If the differential equation $k_{2} k_{1}{ }^{\prime}-k_{1} k_{2}{ }^{\prime}-k_{3}\left(k_{1}{ }^{2}+k_{2}{ }^{2}\right)-\Omega^{\prime}\left(k_{1}{ }^{2}+k_{2}{ }^{2}\right)=0$ is satisfied along $\alpha$ with $k_{1}, k_{2} \neq 0$, then $\alpha$ is a normal curve.

Proof. Before starting the proofs of items, we need some preparation. Let $\alpha=\alpha(s)$ be the unit speed parametrization of the trajectory with $\kappa \neq 0$. One can easily find the equation

$$
\left(k_{1}(s) \sqrt{\left.{k_{1}{ }^{2}(s)+k_{2}{ }^{2}(s)}\right)}\langle\alpha(s), \mathbf{T}(s)\rangle+\left(k_{2}(s)\left(k_{3}(s)+\Omega^{\prime}(s)\right)-k_{1}{ }^{\prime}(s)\right)\langle\alpha(s), \mathbf{N}(s)\rangle+\left(-k_{1}(s)\left(k_{3}(s)+\Omega^{\prime}(s)\right)-k_{2}{ }^{\prime}(s)\right)\langle\alpha(s), \mathbf{B}(s)\rangle=0\right.
$$

by differentiating the equation (3.2) and using the relations between the PAF apparatus and Serret-Frenet apparatus. We can obtain the equation

$$
\left(-k_{1}(s) k_{2}(s) \sqrt{{k_{1}}^{2}(s)+k_{2}{ }^{2}(s)}\right)\langle\alpha(s), \mathbf{T}(s)\rangle+\left(k_{2}(s) k_{1}{ }^{\prime}(s)-k_{1}(s) k_{2}{ }^{\prime}(s)-k_{3}\left(k_{1}{ }^{2}(s)+k_{2}{ }^{2}(s)\right)-\Omega^{\prime}(s)\left(k_{1}{ }^{2}(s)+k_{2}{ }^{2}(s)\right)\right)\langle\alpha(s), \mathbf{N}(s)\rangle=0
$$

if we apply necessary operations to the equations (3.2) and (3.4) side by side. Note that the equation (3.5) plays an important role in the rest of the proof. Now we can discuss the items: 
1. Assume that $\alpha$ is a normal curve. Then its position vector always lies on its normal plane. So,

$$
\langle\alpha(s), \mathbf{T}(s)\rangle=0
$$

for all the values $s$ of the arc-length parameter. Taking into account of the equation (3.5), we obtain

$$
\left(k_{2}(s) k_{1}{ }^{\prime}(s)-k_{1}(s) k_{2}{ }^{\prime}(s)-k_{3}\left(k_{1}{ }^{2}(s)+k_{2}{ }^{2}(s)\right)-\Omega^{\prime}(s)\left(k_{1}{ }^{2}(s)+k_{2}{ }^{2}(s)\right)\right)\langle\alpha(s), \mathbf{N}(s)\rangle=0
$$

for all $s$. On the other hand, differentiating the equation (3.6) yields the following:

$$
\forall s,\langle\alpha(s), \mathbf{N}(s)\rangle=-\frac{1}{\kappa(s)} \neq 0
$$

From the equations (3.7) and (3.8), we conclude

$$
k_{2} k_{1}{ }^{\prime}-k_{1} k_{2}{ }^{\prime}-k_{3}\left(k_{1}{ }^{2}+k_{2}{ }^{2}\right)-\Omega^{\prime}\left(k_{1}{ }^{2}+k_{2}{ }^{2}\right)=0
$$

and finish the proof of this item.

2. Assume that the differential equation $k_{2} k_{1}{ }^{\prime}-k_{1} k_{2}{ }^{\prime}-k_{3}\left(k_{1}{ }^{2}+k_{2}{ }^{2}\right)-\Omega^{\prime}\left(k_{1}{ }^{2}+k_{2}{ }^{2}\right)=0$ is satisfied along $\alpha$ with $k_{1}, k_{2} \neq 0$. From the equation (3.5), we can write

$$
\forall s, \quad\left(-k_{1}(s) k_{2}(s) \sqrt{k_{1}^{2}(s)+k_{2}^{2}(s)}\right)\langle\alpha(s), \mathbf{T}(s)\rangle=0 .
$$

Since $k_{1}, k_{2} \neq 0$, we obtain

$$
\forall s,\langle\alpha(s), \mathbf{T}(s)\rangle=0
$$

which means that $\alpha=\alpha(s)$ is a normal curve.

It is well known that a unit speed curve in $E^{3}$ is a planar curve if and only if the torsion vanishes along this curve (see [14]). Considering this information and the equation $(2.12)_{3}$, the following corollary can be given according to PAF without proof.

Corollary 3.4. In $E^{3}$, assume that a point particle of constant mass moves on its trajectory having non-vanishing angular momentum. Let $\alpha=\alpha(s)$ be the unit speed parametrization of the trajectory with $\kappa \neq 0$. In this case, $\alpha$ is a planar curve if and only if $k_{3}=-\Omega^{\prime}$.

Another well-known class of curves is the class of general helices. In $E^{3}$, a unit speed curve $\alpha=\alpha(s)$ is a general helix if the unit tangent vector of this curve makes a constant angle $\theta$ with a fixed unit vector $\mathbf{u}$; namely,

$$
\langle\mathbf{T}(s), \mathbf{u}\rangle=\cos \theta
$$

for all the $s$ values of parameter. Also, in $E^{3}$, the necessary and sufficient condition for the curve with $\kappa \neq 0$ to be general helix is that ratio of torsion to curvature is constant (see [19]). In the light of this information, we can give the following corollary according to PAF.

Corollary 3.5. In $E^{3}$, assume that a point particle of constant mass moves on its trajectory having non-vanishing angular momentum. Let $\alpha=\alpha(s)$ be the unit speed parametrization of the trajectory with $\kappa \neq 0$. In this case, $\alpha$ is a general helix if and only if

$$
\frac{k_{3}+\Omega^{\prime}}{\sqrt{k_{1}^{2}+k_{2}^{2}}}=\text { constant }
$$

Proof. Let the trajectory $\alpha=\alpha(s)$ be general helix in 3-dimensional Euclidean space $E^{3}$. Then, we have a fixed unit vector $\mathbf{u}$ and a constant angle $\theta$ satisfying

$$
\langle\mathbf{T}, \mathbf{u}\rangle=\cos \theta
$$

from the definition. By differentiating this equation we obtain

$$
\left\langle\mathbf{T}^{\prime}, \mathbf{u}\right\rangle=\left\langle k_{1} \mathbf{M}+k_{2} \mathbf{Y}, \mathbf{u}\right\rangle=\left\langle\sqrt{{k_{1}}^{2}+k_{2}{ }^{2}} \cos \Omega \mathbf{M}+\sqrt{{k_{1}}^{2}+k_{2}{ }^{2}} \sin \Omega \mathbf{Y}, \mathbf{u}\right\rangle=\sqrt{{k_{1}}^{2}+k_{2}{ }^{2}}\langle\cos \Omega \mathbf{M}+\sin \Omega \mathbf{Y}, \mathbf{u}\rangle=0
$$

and so

$$
\langle\cos \Omega \mathbf{M}+\sin \Omega \mathbf{Y}, \mathbf{u}\rangle=0 .
$$

Since $\mathbf{T} \wedge(\cos \Omega \mathbf{M}+\sin \Omega \mathbf{Y})=-\sin \Omega \mathbf{M}+\cos \Omega \mathbf{Y}$, the unit vectors $\mathbf{T}, \cos \Omega \mathbf{M}+\sin \Omega \mathbf{Y},-\sin \Omega \mathbf{M}+\cos \Omega \mathbf{Y}$ compose a right-handed orthonormal system. Then, we can conclude $\mathbf{u} \in \operatorname{Sp}\{\mathbf{T},-\sin \Omega \mathbf{M}+\cos \Omega \mathbf{Y}\}$. Hence

$$
\mathbf{u}=\cos \theta \mathbf{T}+\sin \theta(-\sin \Omega \mathbf{M}+\cos \Omega \mathbf{Y}) .
$$


By differentiation we get

$$
\begin{aligned}
0 & =\cos \theta \mathbf{T}^{\prime}+\sin \theta(-\sin \Omega \mathbf{M}+\cos \Omega \mathbf{Y})^{\prime} \\
& =\left(k_{1} \sin \Omega-k_{2} \cos \Omega\right) \mathbf{T}+\left(k_{1} \cos \theta-\Omega^{\prime} \sin \theta \cos \Omega-k_{3} \sin \theta \cos \Omega\right) \mathbf{M}+\left(k_{2} \cos \theta-\Omega^{\prime} \sin \Omega \sin \theta-k_{3} \sin \Omega \sin \theta\right) \mathbf{Y} .
\end{aligned}
$$

This last equation yields the equation system

$$
\begin{aligned}
k_{1} \sin \Omega-k_{2} \cos \Omega & =0 \\
k_{1} \cos \theta-\Omega^{\prime} \sin \theta \cos \Omega-k_{3} \sin \theta \cos \Omega & =0 \\
k_{2} \cos \theta-\Omega^{\prime} \sin \Omega \sin \theta-k_{3} \sin \Omega \sin \theta & =0 .
\end{aligned}
$$

Due to the equation $(2.12)_{4}$, the first equation of this system is satisfied always. Let us consider the second and third equations together. Then, we can write

$$
\begin{aligned}
& k_{1} \cos \theta=\sin \theta \cos \Omega\left(k_{3}+\Omega^{\prime}\right) \\
& k_{2} \cos \theta=\sin \theta \sin \Omega\left(k_{3}+\Omega^{\prime}\right) .
\end{aligned}
$$

Applying necessary operations these two equations, we obtain

$$
\cos ^{2} \theta\left(k_{1}^{2}+k_{2}^{2}\right)=\sin ^{2} \theta\left(k_{3}+\Omega^{\prime}\right)^{2}
$$

and so

$$
\frac{k_{3}+\Omega^{\prime}}{\sqrt{k_{1}^{2}+k_{2}^{2}}}=\cot \theta=\text { constant }
$$

On the contrary, suppose that $\cot \theta=\frac{k_{3}+\Omega^{\prime}}{\sqrt{k_{1}{ }^{2}+k_{2}{ }^{2}}}$. In this case, we can write

$$
\sqrt{k_{1}^{2}+k_{2}^{2}} \cos \theta-\left(k_{3}+\Omega^{\prime}\right) \sin \theta=0
$$

Differentiating the vector $\mathbf{u}=\cos \theta \mathbf{T}+\sin \theta(-\sin \Omega \mathbf{M}+\cos \Omega \mathbf{Y})$ and using the equalities $k_{1}=\sqrt{{k_{1}}^{2}+k_{2}^{2}} \cos \Omega$ and $k_{2}=\sqrt{k_{1}^{2}+k_{2}^{2}} \sin \Omega$, we can easily obtain

$\mathbf{u}^{\prime}=\left(k_{1} \sin \Omega-k_{2} \cos \Omega\right) \mathbf{T}+\left(\sqrt{{k_{1}}^{2}+k_{2}{ }^{2}} \cos \Omega \cos \theta-\sin \theta \cos \Omega\left(k_{3}+\Omega^{\prime}\right)\right) \mathbf{M}+\left(\sqrt{k_{1}{ }^{2}+k_{2}{ }^{2}} \sin \Omega \cos \theta-\sin \theta \sin \Omega\left(k_{3}+\Omega^{\prime}\right)\right) \mathbf{Y}$.

This gives us the following

$$
\mathbf{u}^{\prime}=\left(\sqrt{k_{1}^{2}+k_{2}^{2}} \cos \theta-\left(k_{3}+\Omega^{\prime}\right) \sin \theta\right)(\cos \Omega \mathbf{M}+\sin \Omega \mathbf{Y}) .
$$

Substituting the equation (3.9) in the last equation yields

$$
\mathbf{u}^{\prime}=0
$$

This means that $\mathbf{u}$ is a constant vector. Thus the proof is completed.

\section{Slant helical trajectories according to PAF}

Similar to general helix, the slant helix was defined in [20] as a curve whose principal normal vector makes a constant angle with a fixed direction in $E^{3}$. That is, if a unit speed curve $\alpha=\alpha(s)$ is a slant helix, then there exist a constant angle $\gamma$ and a fixed unit vector $\mathbf{w}$ satisfying

$$
\langle\mathbf{N}(s), \mathbf{w}\rangle=\cos \gamma
$$

for all the $s$ values of parameter. Throughout the study, we refer it classic slant helix by following the known terminology. The characterization for classic slant helices is given by the equation

$$
\frac{\kappa^{2}}{\left(\kappa^{2}+\tau^{2}\right)^{3 / 2}}\left(\frac{\tau}{\kappa}\right)^{\prime}=\text { constant }
$$

in [20]. After this study, several kind of slant helices have been defined and studied (see [21]-[24] for details). In this section, we define and consider the slant helical trajectories according to PAF and investigate some special cases of them. Note that similar methods and approaches, given in [25], will be followed in this section.

Firstly, we define M-PAF spherical image of the trajectory. We give this spherical image since it is an important part of the characterization of our slant helical trajectories. The remaining PAF spherical images can be topic of a different study of researchers interested.

Definition 4.1. In $E^{3}$, assume that a point particle of constant mass moves on its trajectory having non-vanishing angular momentum. Let $\alpha=\alpha(s)$ be the unit speed parametrization of the trajectory with $\kappa \neq 0$. If we move the second vector field $\mathbf{M}$ of PAF to the center $O$ of the unit sphere $S^{2}$, we get a curve which $\mathbf{M}(s)$ draws on $S^{2}$. We call this curve as $\mathbf{M}-P A F$ spherical image of the trajectory $\alpha=\alpha(s)$ and show it with $\xi_{M}$. 
For M-PAF spherical image of the trajectory $\alpha=\alpha(s)$

$$
\xi_{M}(s)=\mathbf{M}(s)
$$

can be written. If we differentiate this equation with respect to $s$, we obtain

$$
\begin{aligned}
\xi^{\prime}{ }_{M}(s)= & -k_{1}(s) \mathbf{T}(s)+k_{3}(s) \mathbf{Y}(s) \\
\xi^{\prime \prime}{ }_{M}(s)= & {\left[-k_{1}{ }^{\prime}(s)-k_{2}(s) k_{3}(s)\right] \mathbf{T}(s)-\left[k_{1}{ }^{2}(s)+k_{3}{ }^{2}(s)\right] \mathbf{M}(s)+\left[-k_{1}(s) k_{2}(s)+k_{3}{ }^{\prime}(s)\right] \mathbf{Y}(s) } \\
\xi^{\prime \prime \prime}{ }_{M}(s)= & {\left[-k_{1}{ }^{\prime \prime}(s)-k_{2}{ }^{\prime}(s) k_{3}(s)-2 k_{3}{ }^{\prime}(s) k_{2}(s)+k_{1}(s)\left(k_{1}{ }^{2}(s)+k_{2}{ }^{2}(s)+k_{3}{ }^{2}(s)\right)\right] \mathbf{T}(s)-3\left[k_{1}(s) k_{1}{ }^{\prime}(s)+k_{3}(s) k_{3}{ }^{\prime}(s)\right] \mathbf{M}(s) } \\
& +\left[k_{3}{ }^{\prime \prime}(s)-k_{2}{ }^{\prime}(s) k_{1}(s)-2 k_{1}{ }^{\prime}(s) k_{2}(s)-k_{3}(s)\left(k_{1}{ }^{2}(s)+k_{2}{ }^{2}(s)+k_{3}{ }^{2}(s)\right)\right] \mathbf{Y}(s) .
\end{aligned}
$$

These equations give us the curvature $\kappa_{M}$ and the torsion $\tau_{M}$ of $\xi_{M}$ as follows:

$$
\begin{aligned}
\kappa_{M}(s) & =\frac{\left\|\xi_{M}^{\prime}(s) \wedge \xi^{\prime \prime}{ }_{M}(s)\right\|}{\left\|\xi^{\prime}(s)\right\|^{3}}=\sqrt{1+\left(\zeta_{M}(s)\right)^{2}} \\
\tau_{M}(s) & =\frac{\left\langle\xi_{M}^{\prime}(s) \wedge \xi^{\prime \prime}{ }_{M}(s), \xi^{\prime \prime \prime}{ }_{M}(s)\right\rangle}{\left\|\xi^{\prime}{ }_{M}(s) \wedge \xi^{\prime \prime}{ }_{M}(s)\right\|^{2}}=\frac{\zeta_{M}^{\prime}(s)}{\left(1+\left(\zeta_{M}(s)\right)^{2}\right)\left(k_{1}{ }^{2}(s)+k_{3}{ }^{2}(s)\right)^{11 / 2}}
\end{aligned}
$$

where

$$
\zeta_{M}(s)=\left(\frac{k_{3}{ }^{\prime} k_{1}-k_{1}{ }^{\prime} k_{3}-k_{2}\left(k_{1}{ }^{2}+k_{3}{ }^{2}\right)}{\left(k_{1}{ }^{2}+k_{3}{ }^{2}\right)^{3 / 2}}\right)(s) .
$$

Now, we return to slant helices and define the slant helical trajectories having non-vanishing angular momentum according to PAF as in the next definition.

Definition 4.2. In $E^{3}$, assume that a point particle of constant mass moves on its trajectory having non-vanishing angular momentum. Let $\alpha=\alpha(s)$ be the unit speed parametrization of the trajectory with $\kappa \neq 0$. The trajectory $\alpha=\alpha(s)$ is called a slant helical trajectory (according to PAF) if the second vector field $\mathbf{M}$ of PAF makes a constant angle with a fixed direction.

As a result of this definition, if the trajectory $\alpha=\alpha(s)$ is a slant helical trajectory according to PAF, then there exist a constant angle $\beta$ and a fixed unit vector $\mathbf{g}$ satisfying

$$
\langle\mathbf{M}(s), \mathbf{g}\rangle=\cos \beta
$$

for all the $s$ values of parameter.

Theorem 4.3. In $E^{3}$, assume that a point particle of constant mass moves on its trajectory having non-vanishing angular momentum. Let $\alpha=\alpha(s)$ be the unit speed parametrization of the trajectory with $\kappa \neq 0$ and $\left(k_{1}(s), k_{3}(s)\right) \neq(0,0)$. In that case, $\alpha$ is a slant helical trajectory according to PAF if and only if the function, given in the equation (4.3), is a constant function.

Proof. Let the trajectory $\alpha=\alpha(s)$ with $\kappa \neq 0$ and $\left(k_{1}(s), k_{3}(s)\right) \neq(0,0)$ be slant helical trajectory according to PAF in 3-dimensional Euclidean space $E^{3}$. In that case, from the Definition 4.2, the second vector field $\mathbf{M}$ of PAF makes a constant angle with a fixed direction. Therefore, $\mathbf{M}-\mathrm{PAF}$ spherical image $\xi_{M}$ of the trajectory $\alpha=\alpha(s)$ is part of a circle. In other words, it has constant curvature and zero torsion. As we mentioned earlier, the curvature $\kappa_{M}$ and the torsion $\tau_{M}$ of $\xi_{M}$ are as in the equations (4.1) and (4.2). Using the information of constant curvature and zero torsion, we can immediately conclude $\zeta_{M}(s)=$ constant.

On the contrary, assume that $\zeta_{M}(s)=$ constant. In that case, it is very easy to see that $\kappa_{M}(s)=$ constant and $\tau_{M}=0$. Thus, M-PAF spherical image $\xi_{M}$ of the trajectory $\alpha=\alpha(s)$ is part of a circle. This means that $\mathbf{M}$ makes constant angle with a fixed direction and the proof is completed.

Corollary 4.4. Let the trajectory $\alpha=\alpha(s)$ be a rectifying curve with $\kappa, k_{1} \neq 0$. Then, $\alpha$ is a slant helical trajectory according to PAF if and only if

$$
\left(\frac{k_{1}^{2}}{\left(k_{1}^{2}+k_{3}^{2}\right)^{3 / 2}}\left(\frac{k_{3}}{k_{1}}\right)^{\prime}\right)(s)
$$

is a constant function.

Proof. Let the trajectory $\alpha=\alpha(s)$, which is a rectifying curve with $\kappa, k_{1} \neq 0$, be a slant helical trajectory according to PAF. Since $\alpha=\alpha(s)$ is a rectifying curve we obtain $k_{2}=0$ according to Theorem 3.1. If $k_{2}=0$ is substituted in the equation (4.3), we get this equation as

$$
\zeta_{M}(s)=\left(\frac{k_{1}^{2}}{\left(k_{1}^{2}+k_{3}^{2}\right)^{3 / 2}}\left(\frac{k_{3}}{k_{1}}\right)^{\prime}\right)(s) .
$$

Then, Theorem 4.3 finishes the first part of the proof. The other part of the proof can be completed in a similar way.

Corollary 4.5. Let the trajectory $\alpha=\alpha(s)$ be a rectifying curve with $\kappa, k_{1} \neq 0$. Then, $\alpha$ is a slant helical trajectory according to PAF if and only if $\alpha=\alpha(s)$ is a classic slant helix. 
Proof. Let the trajectory $\alpha=\alpha(s)$, which is a rectifying curve with $\kappa, k_{1} \neq 0$, be a slant helical trajectory according to PAF. Due to the Theorem 3.1, we obtain $k_{2}=0$ for rectifying curve $\alpha=\alpha(s)$. Considering $k_{2}=0$, we get $\sin \Omega=0$ and so $k_{3}=\tau, k_{1}= \pm \kappa$. Let us substitute this information in the equation (4.4) and remember the characterization of slant helices given in the beginning of this section. Then, Corollary 4.4 finishes the first part of the proof. In a similar way, one can easily complete the other part of the proof.

Corollary 4.6. Let the trajectory $\alpha=\alpha(s)$ be an osculating curve with $\kappa, k_{3} \neq 0$. Then, $\alpha$ is a slant helical trajectory according to PAF if and only if

$$
\left(\frac{k_{2}}{k_{3}}\right)(s)
$$

is a constant function.

Proof. Let the trajectory $\alpha=\alpha(s)$, which is an osculating curve with $\kappa, k_{3} \neq 0$, be a slant helical trajectory according to PAF. Since $\alpha=\alpha(s)$ is an osculating curve we obtain $k_{1}=0$ according to Theorem 3.2. If $k_{1}=0$ is substituted in the equation (4.3), we get this equation as

$$
\zeta_{M}(s)=\left(-\frac{k_{2}}{k_{3}}\right)(s)
$$

In that case, Theorem 4.3 finishes the first part of the proof. The other part of the proof can be completed in a similar way.

Corollary 4.7. Let the trajectory $\alpha=\alpha(s)$ be an osculating curve with $\kappa, k_{3} \neq 0$. Then, $\alpha$ is a slant helical trajectory according to PAF if and only if $\alpha=\alpha(s)$ is a general helix.

Proof. Let the trajectory $\alpha=\alpha(s)$, which is an osculating curve with $\kappa, k_{3} \neq 0$, be a slant helical trajectory according to PAF. Due to the Theorem 3.2, we obtain $k_{1}=0$ for osculating curve $\alpha=\alpha(s)$. Considering $k_{1}=0$, we get $\cos \Omega=0$ and so $k_{3}=\tau, k_{2}= \pm \kappa$. Let us substitute this information in the equation (4.5) and remember the characterization of general helices given in the previous section. Then, Corollary 4.6 finishes the first part of the proof. In a similar way, one can easily complete the other part of the proof.

Now, we will discuss on the determination of the fixed direction (helix axis) for a slant helical trajectory according to PAF. Assume that a point particle of constant mass moves on a slant helical trajectory (according to PAF) having non-vanishing angular momentum in $E^{3}$. Let $\alpha=\alpha(s)$ be the unit speed parametrization of this trajectory with $\kappa \neq 0$. In this case, there exist a constant angle $\beta$ and a fixed unit vector $\mathbf{g}$ satisfying

$$
\langle\mathbf{M}, \mathbf{g}\rangle=\cos \beta=\lambda_{2}
$$

where $\mathbf{g}=\lambda_{1} \mathbf{T}+\lambda_{2} \mathbf{M}+\lambda_{3} \mathbf{Y}$. From the last equation, we get

$$
\left\langle-k_{1} \mathbf{T}+k_{3} \mathbf{Y}, \mathbf{g}\right\rangle=0
$$

by means of differentiation with respect to $s$. This time, let us differentiate the vector $\mathbf{g}$. Then we get

$$
\left(\lambda_{1}{ }^{\prime}-\lambda_{2} k_{1}-\lambda_{3} k_{2}\right) \mathbf{T}+\left(\lambda_{1} k_{1}-\lambda_{3} k_{3}\right) \mathbf{M}+\left(\lambda_{3}{ }^{\prime}+\lambda_{1} k_{2}+\lambda_{2} k_{3}\right) \mathbf{Y}=0 .
$$

This yields the following equation system:

$$
\begin{array}{r}
\lambda_{1}{ }^{\prime}-\lambda_{2} k_{1}-\lambda_{3} k_{2}=0 \\
\lambda_{1} k_{1}-\lambda_{3} k_{3}=0 \\
\lambda_{3}{ }^{\prime}+\lambda_{1} k_{2}+\lambda_{2} k_{3}=0 .
\end{array}
$$

Here, we will follow similar steps given in [25] to solve this system. If we write $\lambda_{1}=\frac{k_{3}}{k_{1}} \lambda_{3}, k_{1}(s) \neq 0$ in the equations (4.7) $)_{1}$ and (4.7) $)_{3}$ and multiply (4.7) 1 with $\frac{k_{3}}{k_{1}}$, we get the differential equation

$$
\left(1+\left(\frac{k_{3}}{k_{1}}\right)^{2}\right) \lambda_{3}{ }^{\prime}+\frac{k_{3}}{k_{1}}\left(\frac{k_{3}}{k_{1}}\right)^{\prime} \lambda_{3}=0
$$

One can find the general solution $\lambda_{3}=\mu \frac{k_{1}}{\sqrt{k_{1}{ }^{2}+k_{3}{ }^{2}}}$ of this differential equation where $\mu$ is the constant of integration. Then, it is not difficult to obtain $\lambda_{1}=\mu \frac{k_{3}}{\sqrt{k_{1}^{2}+k_{3}^{2}}}$ from the relation $\lambda_{1}=\frac{k_{3}}{k_{1}} \lambda_{3}$. Since the vector $\mathbf{g}=\mu \frac{k_{3}}{\sqrt{k_{1}{ }^{2}+k_{3}^{2}}} \mathbf{T}+\cos \beta \mathbf{M}+\mu \frac{k_{1}}{\sqrt{k_{1}{ }^{2}+k_{3}{ }^{2}}} \mathbf{Y}$ is taken as a unit vector, we can derive the integration constant as $\mu= \pm \sin \beta$. So,

$$
\mathbf{g}= \pm \frac{k_{3}}{\sqrt{k_{1}^{2}+k_{3}^{2}}} \sin \beta \mathbf{T}+\cos \beta \mathbf{M} \pm \frac{k_{1}}{{\sqrt{k_{1}^{2}+k_{3}^{2}}}^{2}} \sin \beta \mathbf{Y}
$$

can be written. Finally, we must determine the constant angle $\beta$. By differentiating the equation (4.6) with respect to arc-length parameter $s$ of the trajectory,

$$
\left\langle\left(-k_{1}{ }^{\prime}-k_{2} k_{3}\right) \mathbf{T}+\left(-k_{1}{ }^{2}-k_{3}{ }^{2}\right) \mathbf{M}+\left(k_{3}{ }^{\prime}-k_{1} k_{2}\right) \mathbf{Y}, \pm \frac{k_{3}}{\sqrt{k_{1}{ }^{2}+k_{3}^{2}}} \sin \beta \mathbf{T}+\cos \beta \mathbf{M} \pm \frac{k_{1}}{\sqrt{k_{1}^{2}+k_{3}^{2}}} \sin \beta \mathbf{Y}\right\rangle=0
$$


is obtained. So, we have

$$
\pm \sin \beta\left(\frac{k_{1} k_{3}{ }^{\prime}-k_{3} k_{1}{ }^{\prime}-k_{2} k_{1}{ }^{2}-k_{2} k_{3}{ }^{2}}{\sqrt{k_{1}{ }^{2}+k_{3}{ }^{2}}}\right)-\cos \beta\left(k_{1}{ }^{2}+k_{3}{ }^{2}\right)=0 .
$$

This yields the following:

$$
\tan \beta= \pm \frac{\left(k_{1}^{2}+k_{3}^{2}\right)^{3 / 2}}{k_{1} k_{3}{ }^{\prime}-k_{3} k_{1}{ }^{\prime}-k_{2} k_{1}^{2}-k_{2} k_{3}^{2}} .
$$

In the light of the above information, one can easily find $\beta$ and determine the fixed direction generated by the constant vector $\mathbf{g}$ (see $\mathbf{g}$ is constant) for the slant helical trajectory according to PAF.

\section{Conclusion}

Since there is a very close relation between a moving point particle of constant mass and the trajectory of it, moving frames adapted to the trajectories are very useful tools for studying the kinematics theory. Considering this relation, a new moving frame, which we call it PAF for short, is introduced for the trajectories with non-vanishing angular momentum in this study. Then, some basic topics are investigated by means of PAF. It may be useful for the researchers studying on modern robotics in their observation environment. Furthermore, we expect that it will be one of the preferred tools for discussing many topics of kinematics and differential geometry.

A natural question is to investigate the special trajectories generated by the TM, TY, MY and TMY - Smarandache curves according to PAF in Euclidean 3-space. We leave that as a future project.

\section{References}

[1] K. Taşköprü, M. Tosun, Smarandache curves on $S^{2}$, Bol. da Soc. Parana. de Mat., 32(1) (2014), 51-59.

[2] K. Eren, H. H. Kösal, Evolution of space curves and the special ruled surfaces with modified orthogonal frame, AIMS Math., 5(3) (2020), 2027-2039.

[3] Ö. G. Yıldız, M. Akyiğit, M. Tosun, On the trajectory ruled surfaces of framed base curves in the Euclidean space, Math. Methods Appl. Sci., 1-8, (2020), https://doi.org/10.1002/mma.6267

[4] B. Y. Chen, When does the position vector of a space curve always lie in its rectifying plane?, Am. Math. Mon., 110(2) (2003), 147-152.

[5] K. İlarslan, E. Nešović, Some characterizations of osculating curves in the Euclidean spaces, Demonstr. Math., 41(4) (2008), 931-939.

[6] Z. Bozkurt, İ. Gök, O. Z. Okuyucu, F. N. Ekmekci, Characterizations of rectifying, normal and osculating curves in three dimensional compact Lie groups, Life Sci. J., 10(3) (2013), 819-823.

[7] R. L. Bishop, There is more than one way to frame a curve, Am. Math. Mon., 82 (1975), 246-251.

[8] S. Yilmaz, M. Turgut, A new version of Bishop frame and an application to spherical images, J. Math. Anal. Appl., 371(2) (2010), 764-776.

[9] M. A. Soliman, N. H. Abdel-All, R. A. Hussien, T. Youssef, Evolution of space curves using Type-3 Bishop frame, Caspian J. Math. Sci. 8(1) (2019), $58-73$.

[10] M. Dede, C. Ekici, H. Tozak, Directional tubular surfaces, Int. J. Algebra, 9(12) (2015), 527-535.

[11] G. Y. Şentürk, S. Yüce, Bertrand offsets of ruled surfaces with Darboux Frame, Results Math., 72(3) (2017), $1151-1159$.

[12] B. Uzunoğlu, İ. Gök, Y. Yaylı, A new approach on curves of constant precession, Appl. Math. Comput., 275 (2016), $317-323$.

[13] O. Keskin, Y. Yayli, An application of N-Bishop frame to spherical images for direction curves, Int. J. Geom. Methods Mod. Phys., 14(11) (2017), 1750162.

[14] T. Shifrin, Differential Geometry: A First Course in Curves and Surfaces, University of Georgia, Preliminary Version, 2008.

[15] A. Menninger, Characterization of the slant helix as successor curve of the general helix, Int. Electron. J. Geom., 7(2) (2014), 84-91.

[16] J. Casey, Siacci's resolution of the acceleration vector for a space curve, Meccanica, 46 (2011), 471-476.

[17] K. E. Özen, F. S. Dündar, M. Tosun, An alternative approach to jerk in motion along a space curve with applications, J. Theor. Appl. Mech., 57(2) (2019), 435-444.

[18] K. E. Özen, M. Tosun, On the resolution of the acceleration vector according to Bishop frame, Univers. J. Math. Appl., 4(1) (2021), 26-32.

[19] D. J. Struik, Lectures on Classical Differential Geometry, Dover, New-York, 1988.

[20] S. Izumiya, N. Takeuchi, New special curves and developable surfaces, Turk. J. Math. 28 (2004), 153-163.

[21] B. Bükcü, M. K. Karacan. The slant helices according to Bishop frame, Int. J. Comput. Math. Sci., 3 (2009), 67-70.

[22] A. T. Ali, M. Turgut, Some characterizations of slant helices in the Euclidean space E ${ }^{n}$, Hacet. J. Math. Stat. 39(3) (2010), $327-336$.

[23] O. Z. Okuyucu, İ. Gök, Y. Yayl1, N. Ekmekci, Slant helices in three dimensional Lie groups, Appl. Math. Comput., 221 (2013), $672-683$.

[24] P. Lucas, J. A. Ortega-Yagües, Helix surfaces and slant helices in the three-dimensional anti-De Sitter space, RACSAM 111(4) (2017), 1201-1222.

[25] N. Macit, M. Düldül, Relatively normal-slant helices lying on a surface and their characterizations, Hacet. J. Math. Stat., 46(3) (2017), $397-408$. 